\section{Genome Sequence of the Plant Growth Promoting Fungus Serendipita vermifera subsp. bescii: The First Native Strain from North America}

Prasun Ray, ${ }^{1}$ Myoung-Hwan Chi, ${ }^{1}$ Yingqing Guo, ${ }^{1}$ Cindy Chen, ${ }^{2}$ Catherine Adam, ${ }^{2}$ Alan Kuo, ${ }^{2}$ Kurt LaButti, ${ }^{2}$ Anna Lipzen, ${ }^{2}$ Kerrie W. Barry, ${ }^{2}$ Igor V. Grigoriev, ${ }^{2}$ Yuhong Tang, ${ }^{1}$ and Kelly D. Craven ${ }^{1, \dagger}$

${ }^{1}$ Noble Research Institute, LLC, Ardmore, OK 73401

${ }^{2}$ U.S. Department of Energy (DOE) Joint Genome Institute, Walnut Creek, CA 94598

\begin{abstract}
Serendipita vermifera (=Sebacina vermifera) has demonstrated plant growth promoting abilities with little or no host specificity. The full genome sequencing of Serendipita vermifera subsp. bescii, the first North American strain, will provide insight into the mechanisms behind mycorrhizal symbiosis and plant enhancement in a variety of agronomically important crops.
\end{abstract}

The family Serendipitaceae (formerly Sebacinales Group B) are a taxonomically, ecologically, and physiologically diverse group of fungi in the Basidiomycota. While historically recognized as orchid mycorrhizae, recent DNA studies have brought to light both their pandemic distribution and the broad spectrum of mycorrhizal types they form (Oberwinkler et al. 2013). The orchid mycorrhizal fungus Serendipita vermifera (=Sebacina vermifera isolate MAFF 305830) was first isolated from the Australian orchid Cyrtostylis reniformis (Warcup 1988). Research performed with Serendipita vermifera clearly indicates its plant growth promoting abilities in a variety of plants (Ghimire and Craven 2011; Ray and Craven 2016; Ray et al. 2015), demonstrating little or no host specificity. Considering their proven beneficial impact on plant growth and their apparent ubiquity, Serendipitaceae should be considered as a previously hidden, but amenable and effective microbial tool for enhancing plant productivity and stress tolerance. Unfortunately, the agronomic utility of these fungi is currently hampered by the paucity of strains available, the large majority isolated from Australian orchids. It is likely that these Serendipitaceae have been overlooked by classical culture-based approaches as they are notoriously difficult to isolate in culture (Berch et al. 2002; Selosse et al. 2007), a notion supported by studies comparing fungi detected by culture-dependent and -independent approaches, the latter defined by direct amplification of fungal ribosomal DNA ( $\mathrm{rDNA}$ ) from environmental samples. Hence full genome sequencing of Serendipita vermifera subsp. bescii isolated from switchgrass (Panicum virgatum L.), the first such strain from any North American plant (Craven and Ray 2017), will provide novel insight into the phytobiome, genetic and physiological diversity, and the variety of mechanisms employed by these fungi for mycorrhizal symbiosis and crop improvement not only in switchgrass but also other agronomically important crops.

Serendipita vermifera subsp. bescii NFPB0129 was isolated from Noble Research Institute's switchgrass field located in Ardmore, Oklahoma, USA. The genome and transcriptome of this strain were sequenced using the lllumina platform. DNA was extracted using DNeasy Plant Mini Kit (Qiagen Inc., Germantown, MD) and RNA was extracted using Quick-RNA Miniprep (Zymo Research Corp., Irvine, CA) following manufacturer's instruction. One hundred nanograms of DNA was sheared to $300 \mathrm{bp}$ using the Covaris LE220 Focused-Ultrasonicator (Covaris, Inc., Woburn, MA) and size selected using SPRI beads (Beckman Coulter, Brea, CA). The fragments were

${ }^{\dagger}$ Corresponding author: K. D. Craven; E-mail: kdcraven@noble.org

Accepted for publication 18 April 2018.
Funding

This work was supported by the U.S. Department of Energy (DOE) Joint Genome Institute, a DOE Office of Science User Facility, and the Bioenergy Science Center, a U.S. Department of Energy Bioenergy Research Center, through the Office of Biological and Environmental Research in the DOE Office of Science under Contract Number DE-AC02-05CH11231.

\section{Keywords}

agriculture, crop, genomics, microorganism, mycology, rhizosphere and phyllosphere, symbiosis 
Table 1. Summary statistics for Serendipita vermifera subsp. bescii and its close relatives

\begin{tabular}{|c|c|c|c|}
\hline Statistic & $\begin{array}{l}\text { Serendipita vermifera } \\
\text { subsp. bescii NFPB0129 }\end{array}$ & $\begin{array}{c}\text { Serendipita vermifera } \\
\text { MAFF } 305830\end{array}$ & Piriformospora indica \\
\hline Reference & This study & (Kohler et. al. 2015) & (Zuccaro et al. 2011) \\
\hline Isolated from & USA & Australia & India \\
\hline Genome size $(\mathrm{Mb})$ & 36.9 & 38.1 & 25.0 \\
\hline Scaffold number & 827 & 546 & 1,884 \\
\hline Scaffold N50 (kb) & 205 & 319 & 52 \\
\hline Largest scaffold (Mb) & 1.11 & 1.90 & 0.25 \\
\hline $\mathrm{GC}$ contents $(\%)$ & 47.5 & 48.9 & 50.7 \\
\hline Repetitive DNA (\%) & 0.5 & 0.7 & 0.5 \\
\hline Protein coding genes & 14,208 & 15,312 & 11,767 \\
\hline
\end{tabular}

treated with end-repair, A-tailing, and ligation of Illumina compatible adapters (IDT, Inc., San Jose, $\mathrm{CA}$ ) using the KAPA-Illumina library creation kit (KAPA Biosystems, Wilmington, MA). A single transcriptome library was generated by using total RNA from 14-day-old fresh vegetative mycelia growing on modified Melin Norkan's broth (Marx 1969) under static condition at optimum temperature $\left(\sim 24^{\circ} \mathrm{C}\right)$ and $\mathrm{pH}(\sim 7)$. For the transcriptome, cDNA libraries were generated using the Illumina Truseq Stranded RNA LT kit following the manufacturer's instructions. The prepared libraries were quantified and then multiplexed into a pool, to generate a clustered flow cell for sequencing. Sequencing of the flow cell was performed on the Illumina HiSeq2500 sequencer, following a $2 \times 150$ indexed run recipe. Genomic reads were QC-filtered for artifact/process contamination/mitochondrion and assembled with AllPathsLG release version R49403 (Gnerre et al. 2011) to produce a $36.85 \mathrm{Mb}$ assembly in 827 scaffolds. Using the JGI Annotation pipeline, 14,208 gene models were predicted (Grigoriev et al. 2013). Approximately $4 \%$ of the genome was found to be repetitive by kmer analysis (Liu et al. 2013) of the fragment data.

Genome size and predicted number of genes of Serendipita vermifera subsp. bescii NFPB0129 (this study) and Serendipita vermifera (MAFF 305830) (Kohler et al. 2015) are similar to each other, while they are slightly larger than those of Serendipita indica (Zuccaro et al. 2011) (Table 1).

Nucleotide sequence accession numbers. The genome sequences and annotations are available at JGI MycoCosm (Grigoriev et al. 2013) https://genome.jgi.doe.gov/Sebvebe1. This Whole Genome Shotgun project has been deposited at DDBJ/ENA/GenBank under the accession PPET00000000. The version described in this paper is version PPET01000000.

\section{Literature Cited}

Berch, S., Allen, T., and Berbee, M. 2002. Molecular detection, community structure and phylogeny of ericoid mycorrhizal fungi. Plant Soil 244:55-66.

Craven, K. D., and Ray, P. 2017. A symbiont for enhancement of plant performance. United States Patent Application No. US 2017/0360049 A1.

Ghimire, S. R., and Craven, K. D. 2011. Enhancement of switchgrass (Panicum virgatum L.) biomass production under drought conditions by the ectomycorrhizal fungus Sebacina vermifera. Appl. Environ. Microbiol. 77:7063-7067.

Gnerre, S., MacCallum, I., Przybylski, D., Ribeiro, F. J., Burton, J. N., Walker, B. J., Sharpe, T., Hall, G., Shea, T. P., and Sykes, S. 2011. High-quality draft assemblies of mammalian genomes from massively parallel sequence data. Proc. Natl. Acad. Sci. 108:1513-1518.

Grigoriev, I. V., Nikitin, R., Haridas, S., Kuo, A., Ohm, R., Otillar, R., Riley, R., Salamov, A., Zhao, X., and Korzeniewski, F. 2013. MycoCosm portal: Gearing up for 1000 fungal genomes. Nucleic Acids Res. 42:D699-704.

Kohler, A., Kuo, A., Nagy, L. G., Morin, E., Barry, K. W., Buscot, F., Canbäck, B., Choi, C., Cichocki, N., and Clum, A. 2015. Convergent losses of decay mechanisms and rapid turnover of symbiosis genes in mycorrhizal mutualists. Nat. Genet. 47:410-415.

Liu, B., Shi, Y., Yuan, J., Hu, X., Zhang, H., Li, N., Li, Z., Chen, Y., Mu, D., and Fan, W. 2013. Estimation of genomic characteristics by analyzing k-mer frequency in de novo genome projects. arXiv Preprint arXiv:1308.2012v1.
Marx, D. H. 1969. The influence of ectotrophic mycorrhizal fungi on the resistance of pine roots to pathogenic infections. I. Antagonism of mycorrhizal fungi to root pathogenic fungi and soil bacteria. Phytopathology 59:153-163.

Oberwinkler, F., Riess, K., Bauer, R., Selosse, M.-A., Weiß, M., Garnica, S., and Zuccaro, A. 2013. Enigmatic sebacinales. Mycol. Prog. 12:1-27.

Ray, P., and Craven, K. D. 2016. Sebacina vermifera: A unique root symbiont with vast agronomic potential. World J. Microbiol. Biotechnol. 32:16.

Ray, P., Ishiga, T., Decker, S. R., Turner, G. B., and Craven, K. D. 2015. A novel delivery system for the root symbiotic fungus, Sebacina vermifera, and consequent biomass enhancement of low lignin COMT switchgrass lines. BioEnergy Res. 8: 922-933.

Selosse, M. A., Setaro, S., Glatard, F., Richard, F., Urcelay, C., and Weiß, M. 2007. Sebacinales are common mycorrhizal associates of Ericaceae. New Phytol. 174: 864-878.

Warcup, J. 1988. Mycorrhizal associations of isolates of Sebacina vermifera. New Phytol. 110:227-231.

Zuccaro, A., Lahrmann, U., Güldener, U., Langen, G., Pfiffi, S., Biedenkopf, D., Wong, P., Samans, B., Grimm, C., and Basiewicz, M. 2011. Endophytic life strategies decoded by genome and transcriptome analyses of the mutualistic root symbiont Piriformospora indica. PLoS Pathog. 7:e1002290. 